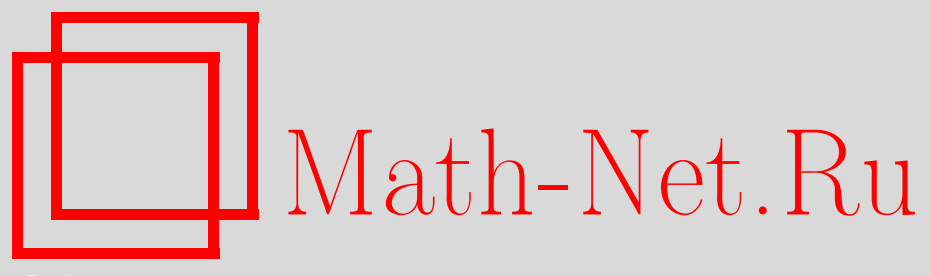

Б. Т. Сапарбаева, Двумерные конечнозонные операторы Шрёдингера с эллиптическими коэффициентами, $M a$ тем. заметки, 2014, том 95, выпуск 5, 798-800

DOI: https://doi.org/10.4213/mzm10447

Использование Общероссийского математического портала Math-Net.Ru подразумевает, что вы прочитали и согласны с пользовательским соглашением http://www . mathnet.ru/rus/agreement

Параметры загрузки:

IP : 54.172 .240 .79

26 апреля 2023 г., 18:25:00

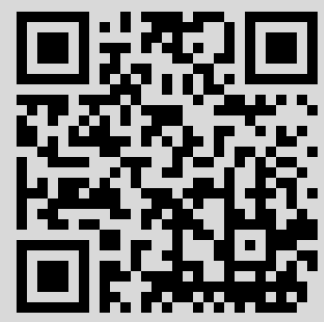




\section{Двумерные конечнозонные операторы Шрёдингера с эллиптическими коэффициентами}

\section{Б. Т. Сапарбаева}

В данной работе мы строим примеры конечнозонных на одном уровне энергии двумерных операторов Шрёдингера, коэффициенты которых выражены через ю-функцию Вейерштрасса, при этом спектральные кривые операторов имеют род больше 1.

Конечнозонные на одном уровне энергии $2 D$ операторы Шрёдингера введены Дубровиным, Кричевером и Новиковым [4]. Такие операторы восстанавливаются по спектральным данным $\left\{\Gamma, q_{1}, q_{2}, \gamma\right\}$, где $\Gamma$ - риманова поверхность, $q_{1}, q_{2}$ - выделенные точки на $\Gamma, \gamma-$ неспециальный дивизор степени $g, g$ - род Г. Существует единственная функция $\psi(z, \bar{z}, P)$, $P \in \Gamma$, которая обладает следующими свойствами:

1) в окрестностях $q_{1}$ и $q_{2}$ имеют место разложения

$$
\begin{aligned}
& \psi=e^{k_{1} z}\left(1+\frac{\xi(z, \bar{z})}{k_{1}}+O\left(\frac{1}{k_{1}^{2}}\right)\right) \\
& \psi=e^{k_{2} \bar{z}}\left(\mu_{0}(z, \bar{z})+\frac{\mu_{1}(z, \bar{z})}{k_{2}}+O\left(\frac{1}{k_{2}^{2}}\right)\right),
\end{aligned}
$$

2) на $\Gamma \backslash\left\{q_{1}, q_{2}\right\}$ функция $\psi$ имеет дивизор полюсов $\gamma$.

Функция $\psi$ называется двухточечной функцией Бейкера-Ахиезера. Функция $\psi$ удовлетворяет уравнению Шрёдингера

$$
H \psi=\left(\frac{\partial^{2}}{\partial z \partial \bar{z}}+\nu(z, \bar{z}) \frac{\partial}{\partial \bar{z}}+u(z, \bar{z})\right) \psi,
$$

где $\nu(z, \bar{z})=-(\partial / \partial z) \ln \mu_{0}, u=-(\partial / \partial \bar{z}) \xi$. Функция Бейкера-Ахиезера имеет вид

$$
\psi(z, \bar{z}, P)=\exp \left\{z \int_{Q}^{P} \Omega_{1}+\bar{z} \int_{Q}^{P} \Omega_{2}\right\} \frac{\theta\left(U_{1} z+U_{2} \bar{z}+f(P)+W\right) \theta(W)}{\theta(f(P)+W) \theta\left(U_{1} z+U_{2} \bar{z}+W\right)},
$$

где $\theta$ - тэта-функция многообразия Якоби спектральной кривой $\Gamma, \Omega_{i}$ - нормированный мероморфный дифференциал с полюсом второго порядка в $q_{i}, f(P)$ - отображение Абеля, $U_{i}, V_{i}, W$ - некоторые векторы, определяемые спектральными данными, $Q$ - фиксированная точка на Г (см. в [4]). При этом

$$
\nu(z, \bar{z})=-\frac{\partial}{\partial z} \ln \frac{\theta\left(U_{1} z+U_{2} \bar{z}+V_{1}+W\right)}{\theta\left(U_{1} z+U_{2} \bar{z}+V_{2}+W\right)}, \quad u(z, \bar{z})=\frac{\partial^{2}}{\partial z \partial \bar{z}} \ln \theta\left(U_{1} z+U_{2} \bar{z}+W\right) .
$$

Пусть $f_{1}, f_{2}$ - мероморфные функции на $Г$ с полюсом в $q_{1}$, а $g_{1}, g_{2}$ - мероморфные функции с полюсом в $q_{2}$. Тогда существуют единственные дифференциальные операторы $L\left(f_{i}\right)$ и $\widetilde{L}\left(g_{i}\right)$ такие, что

$$
\begin{aligned}
L\left(f_{i}\right) \psi=f_{i} \psi, & \widetilde{L}\left(g_{i}\right) \psi=g_{i} \psi, \\
{\left[L\left(f_{1}\right), L\left(f_{2}\right)\right]=0, } & {\left[\widetilde{L}\left(g_{1}\right), \widetilde{L}\left(g_{2}\right)\right]=0 . }
\end{aligned}
$$

Работа выполнена при поддержке гранта Правительства Российской Федерации для государственной поддержки научных исследований (грант № 14.В25.31.0029), а также программы «Динамические системы в геометрии и математической физике» (грант 1431/ГФ2, руководитель И. А. Тайманов.

DOI: $10.4213 / \operatorname{mzm} 10447$ 
Оператор Шрёдингера $H$ удовлетворяет тождеству

$$
\left[H, L\left(f_{i}\right)\right]=B_{i} H, \quad\left[H, \widetilde{L}\left(g_{i}\right)\right]=\widetilde{B}_{i} H,
$$

где $B_{i}$ и $\widetilde{B}_{i}-$ некоторые операторы.

Конечнозонные $2 D$-операторы Шрёдингера изучались во многих работах (см. например [2]-[7]). Основные результаты этой работы - следующие теоремы.

Tеорема 1. Onератор Шрёдингера

$$
H=\frac{\partial^{2}}{\partial z \partial \bar{z}}+a\left(\frac{\sqrt{g_{0}}-\wp^{\prime}(a z+b \bar{z})}{2 \wp(a z+b \bar{z})}\right) \frac{\partial}{\partial \bar{z}}-\frac{b g(g+1) \wp(a z+b \bar{z})}{2 a}
$$

является конечнозонным, где ю - эллиптическая функиия Вейерштрасса, удовлетворяющая уравнению

$$
\left(\wp^{\prime}(z)\right)^{2}=\frac{2 g(g+1)}{a^{2}} \wp(z)^{3}+g_{2} \wp(z)^{2}+g_{1} \wp(z)+g_{0} .
$$

Спектральная кривая оператора $H$ - гиперэллитическая кривая рода $\mathrm{g}$.

Таким образом, для оператора $H$ тэта-функциональные формулы (1) редуцируются к более простым формулам (4). Отметим, что $H$ удовлетворяет тождеству

$$
\left[H,-\frac{\partial^{2}}{\partial z^{2}}+g(g+1) \wp(a z+b \bar{z})\right]=-2 a\left(\frac{\partial}{\partial z}\left(\frac{\sqrt{g_{0}}-\wp^{\prime}(a z+b \bar{z})}{2 \wp(a z+b \bar{z})}\right)\right) H .
$$

Несложно найти другие операторы $L\left(f_{i}\right), \widetilde{L}\left(g_{i}\right)$ для $H$ из $(2),(3)$.

Напомним, что в общем случае потенциал конечнозонного одномерного оператора Шрёдингера $-\partial^{2} / \partial x^{2}+u(x)$ выражается через тэта-функцию спектральной кривой (см. [8]). В то же время существуют конечнозонные операторы с потенциалами, выраженными через ю-функцию Вейерштрасса, например, оператор Ламе

$$
-\frac{\partial^{2}}{\partial x^{2}}+g(g+1) \wp(x)
$$

или оператор Трейбича-Вердне

$$
-\frac{\partial^{2}}{\partial x^{2}}+\sum_{i=0}^{3} a_{i}\left(a_{i}+1\right) \wp\left(x+\omega_{i}\right)
$$

где $\omega_{i}$ - полупериод. Теорема 1 показывает, что аналогичный феномен возможен и в двумерном случае.

Tеорема 2. Onератор Шрёдингера

$$
H=\frac{\partial^{2}}{\partial z \partial \bar{z}}+\frac{7 a \wp^{\prime}(a z+b \bar{z})}{20 g_{2} a^{2}-14 \wp(a z+b \bar{z})} \frac{\partial}{\partial \bar{z}}+\frac{b \wp(a z+b \bar{z})}{2 a}
$$

является конечнозонным, где ю удовлетворяет уравнению

$$
\left(\wp^{\prime}(z)\right)^{2}=-\frac{1}{2 a^{2}} \wp(z)^{3}+g_{2} \wp(z)^{2}-\left(\frac{7 g_{0}}{10 g_{2} a^{2}}+\frac{20 g_{2}^{2} a^{2}}{49}\right) \wp(z)+g_{0} .
$$

Оператор $H$ из теоремы 2 коммутирует с самосопряженным оператором $L_{4}$ по модулю $H$

$$
\left[H, L_{4}\right]=B H,
$$

где

$$
L_{4}=\left(\frac{\partial^{2}}{\partial z^{2}}+\wp(a z+b \bar{z})\right)^{2}-\frac{15}{14} g_{2} a^{2} \wp(a z+b \bar{z})+\frac{3}{8} \wp(a z+b \bar{z})^{2} .
$$

Отметим, что самосопряженные операторы четвертого порядка, коммутирующие с операторами порядка $4 g+2$, изучались в [9].

Автор благодарит А. Е. Миронова за постановку задачи и полезные обсуждения. 


\section{СПИСОК ЦИТИРОВАННОЙ ЛИТЕРАТУРЫ}

[1] Б. А. Дубровин, И. М. Кричевер, С. П. Новиков, Докл. АН СССР, 229:1 (1976), 15-18. [2] А. П. Веселов, С. П. Новиков, Докл. АН СССР, 279:4 (1984), 784-788. [3] П. Г. Гриневич, А.Е. Миронов, С. П. Новиков, УМН, 65:3(393) (2010), 195-196. [4] С. П. Новиков, Функи. анализ и его прил., 8:3 (1974), 54-66. [5] И. М. Кричевер, УМН, 44:2 (1989), 121-184. [6] И. А. Тайманов, ТМФ, 84:1 (1990), 38-45. [7] И. В. Чередник, Докл. АН СССР, 252:5 (1980), 1104-1108. [8] А. Р. Итс, В. Б. Матвеев, ТМФ, 23:1 (1975), 51-68. [9] А. E. Mironov, Invent. Math. (to appear).

Б. Т. Сапарбаева

Поступило

Институт математики им. С. Л. Соболева СО РАН, 29.11.2013

г. Новосибирск

E-mail: sopik2002@mail.ru 